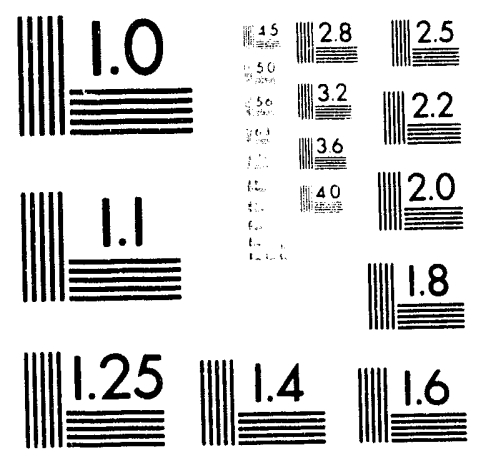



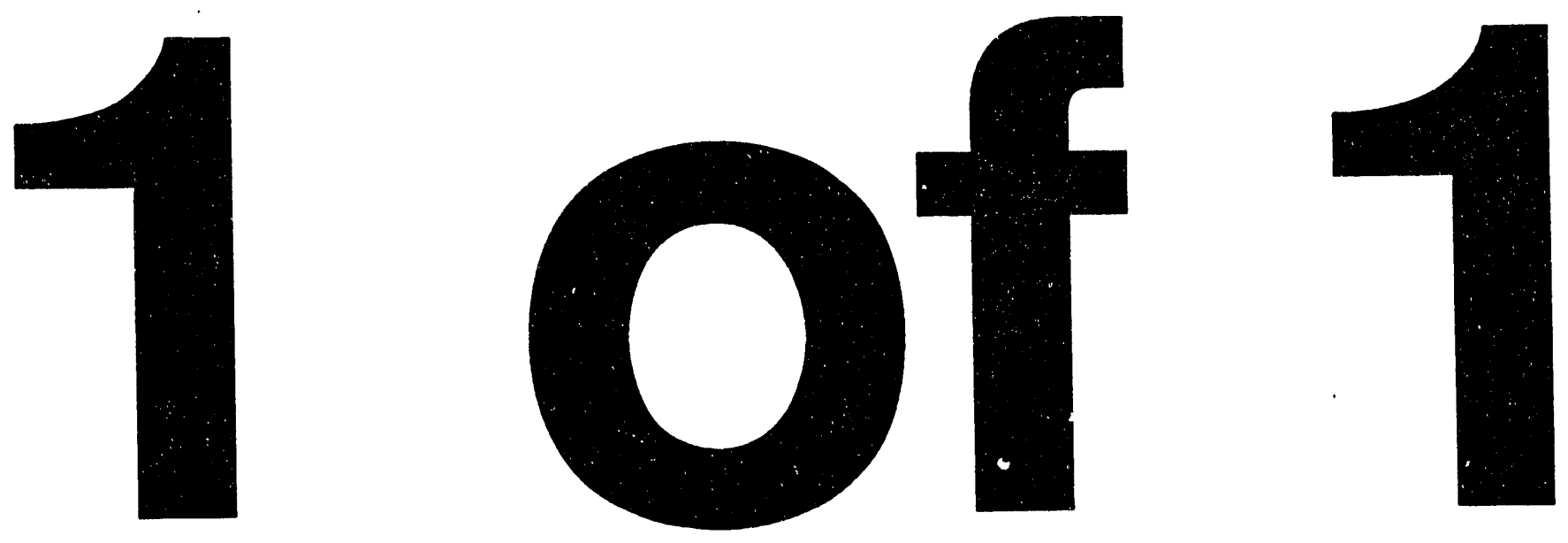

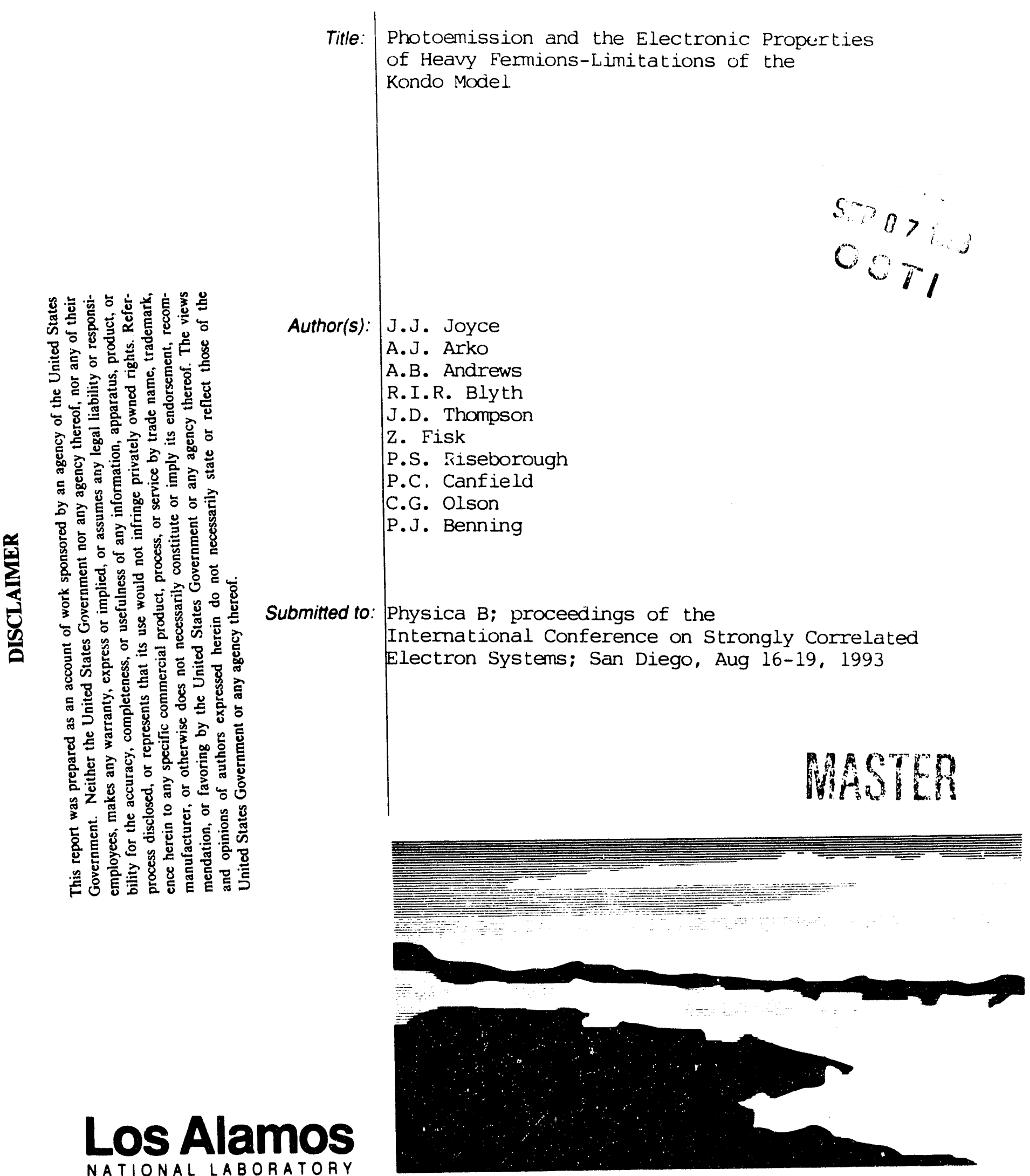

NATIONAL LABORATORY

Los Alamos National Laboratory, an attirmative action/equal oppontunity emplifyer. is operated by the University of California for the US. Department of Energy under contrat W.7405.ENG.36. By acceplance of this article, the publisher recognizes that the US. Government retains a nonexclusive. royalty- tree license to under con publish or reproduce the published torm of this conthbution, or to allow others 10 do so, tor U.S. Governmentist of Energy 


\title{
PHOTOEMISSION AND THE ELECTRONIC PROPERTIES OF HEAVY FERMIONS - LIMITATIONS OF THE KONDO MODEL
}

\author{
J.J. Joyce, A.J.Arko, A.B. Andrews, R.I.R. Blyth, R.J. Bartlett, J.D. Thompson and Z. Fis': \\ Los Alamos National Laboratory, Los Alamos, New Mexico, USA , 87545 \\ P.S. Riseborough \\ Polytechnic Institute of New York, Department of Physics, Brooklyn, NY, ISA 11201 \\ P.C. Canfield, C.G. Olson and P.J. Benning \\ Ames Laboratory USDOE, Ames, Iowa, LSA 50011
}

The electronic properties of $\mathrm{Yb}$-based heavy fermions have been investigated by means of high resolution synchrotron radiation photoemission and compared with predictions of the Kondo model. The $\mathrm{Yb}$ heavy fermion photoemission spectra show massive disagreement with the Kondo model predictions (as calculated within the Gunnarsson-Schonhammer computational method). Moreover, the $\mathrm{Yb}$ heavy fermion photoemission spectra give very strong indications of core-like characteristics and compare favorable to purely divalent $\mathrm{Yb}$ metal and core-like Lu $4 \mathrm{f} \mathrm{levels.} \mathrm{The}$ heavy fermions $\mathrm{YbCu}_{2} \mathrm{Si}_{2}, \mathrm{YbAgCu}_{4}$ and $\mathrm{YbAl}_{3}$ were measured and shown to have lineshapes much broader and deeper in binding energy than predicted by the Kondo model. The lineshape of the bulk component of the $4 \mathrm{f}$ emission for these three heavy fermion materials was compared with that from $\mathrm{Yb}$ metal and the $\mathrm{Lu} 4 \mathrm{f}$ levels in $\mathrm{LuAl}_{3}$, the heavy fermion materials show no substantive spectroscopic differences from simple $4 \mathrm{f}$ levels observed in $\mathrm{Yb}$ metal and $\mathrm{LuAl}_{3}$. Also, the variation with temperature of the $4 \mathrm{f}$ lineshape was measured for $\mathrm{Yb}$ metal and clearly demonstrates that phonon broadening plays a major role in $4 \mathrm{f}$ level lineshape analysis and must be accounted for before considerations of correlated electron resonance effects are presumed to be at work 


\section{Introduction}

The agreement between theory and experiment for the novel electronic properties of heavy fermion materials has long been forged by model calculations, often termed the Kondo model (KM), and the associated Gunnarsson-Schonhammer (GS) [1] and non-crossing approximation (NCA) [2] computational methods. For several years photoelectron spectroscopy (PES) has been called on to provide experimental verification for these model calculations. [3,4] More recently, improvements in 1) the availability of high quality samples; 2) use of high-resolution synchrotron PES; and 3) improvements in the level of sophistication for the GS and NCA methods, have lead to a reexamination of the long accepted agreement between experimental results and model calculations.[5-8] The Kondo model, within the GS and NCA framework, predicts a sharply-peaked, resonance arising from the interaction of f-level and conduction band spin states.[1,2] This socalled Kondo resonance $(K R)$ has a characteristic temperature $\left(T_{K}\right)$ which sets the low energy scale $\left(K_{B} T_{K}\right)$ for the material and determines the energy position and line width which are expected from PES measurements. For low $T_{K}$ heavy fermion materials $\left(T_{K}<200 \mathrm{~K}\right.$ ), the $K R$ should give rise to electronic structures vastly different (much narrower in energy) than a standard band-structure or core-level picture. The $\mathrm{KM}$ becomes more complicated as the $\mathrm{N}=14 \mathrm{f}$-level degeneracy is lifted by crystal field (CEF) and spin orbit (SOS) splittings, which give rise to Kondo resonance sidebands associated with the primar; Kondo resonance. Still, the current level of sophistication in the model calculations can account for these sidebands, and the PES experimental resolution is sufficient to distinguish these Kondo resonance features from standard PES electronic structure as will be shown below. While past PES studies have concentrated primarily on Ce-based heavy fermions [3-5], a great deal of activity over the past two years has focused on Yb-based heavy fermion PES studies.[8-11] Emphasis on Yb-based PES work is driven by the great advantage of having the Kondo resonance on the occupied portion of the density of states (DOS) for $\mathrm{Yb}$ systerns where the KR should be directly observable in PES. Early work on the

heavy fermion $\mathrm{YbA} 33[4,12]$ has recently been followed by three additional studies $[8-10]$ and 
thus represents the largest body of PES Yb-based heavy fermion work. We present PES results for $\mathrm{YbAl} 3, \mathrm{YbAgCu} 4$, and $\mathrm{YbCu} 2 \mathrm{Si} 2$, thus providing a wide range of Kondo temperatures, as well as the non-Kondo systems LuAl3 and $\mathrm{Yb}$ metal providing a base-line for comparison to conventional $4 \mathrm{f}$ materials. The five materials included in this study show PES characteristics indicative of a generalized $4 \mathrm{f}$ photoemission picture which does not require a $\mathrm{KM}$ interpretation for the heavy fermion materials and is fully described by conventional PES analysis methodologies.

\section{Experimental}

Experiments were carried out at the Los Alamos U3C beamline at the National Synchrotron Light Source and the Ames/Montana State ERG/Seya beamline at the Synchrotron Radiation Center. The samples were flux-grown single crystals where possible $\left(\mathrm{YbCu}_{2} \mathrm{Si}_{2}, \mathrm{YbAl}_{3}\right.$, $\mathrm{LuAl}_{3}$ ), slow cooling of stoichiometric melt, sealed in $\mathrm{Ta}$, for $\mathrm{YbAgCu}_{4}$, and UHV in situ evaporated $\mathrm{Yb}$ films for $\mathrm{Yb}$ metal. The samples were cleaved or evaporated in ultra high vacuum with a chamber base pressure of $3-7 \times 10^{-11}$ Torr. Temperature was controlled by placing the sample in direct contact with a cryostat cooled either by liquid helium, liquid nitrogen, or a closed cycle He refrigerator. The experimental resolution was determined to be $45-65 \mathrm{meV}$ at $h v=60 \mathrm{v} Y$ and $\sim 100$ $\mathrm{meV}$ at $\mathrm{h} \nu=102-120 \mathrm{eV}$ for these high resolution experiments. Although the characteristic Kondo and crystal field linewidths are expected to be smaller than this resolution, we show that the natural line width is $\approx 100 \mathrm{meV}$; and thus experimental resolution is more than acequate. This point is established in detail elsewhere. $[5,6]$ The characteristic temperatures of the heavy fermions considered in this work are $\mathrm{YbCu}_{2} \mathrm{Si}_{2}\left(\mathrm{~T}_{\mathrm{K}} \approx 40 \mathrm{~K}\right), \mathrm{YbAl}_{3}\left(\mathrm{~T}_{\mathrm{K}} \approx 400 \mathrm{~K}\right)$ and $\mathrm{YbAgCu}_{4}$ $\left(\mathrm{T}_{\mathrm{K}} \approx 100 \mathrm{~K}\right)$.

\section{Results and Discussion}

In Fig. 1 we show PES results (la) as well as GS calculations (1b) for YbCu2Si2 with the energy reference being the Fermi level. The full valence band taken at a photon energy of $102 \mathrm{eV}$ is shown in (la) consisting of the $\mathrm{Cu} d$ bands $(-5 \mathrm{eV})$, the divalent and trivalent $\mathrm{Yb}$ 4f signal on 
either side of the $\mathrm{Cu} d$ state and also the Si $2 p$ core-level at $+3 \mathrm{eV}$ which arise from second order photons $(204 \mathrm{eV})$. The region of interest for heavy fermion characteristics is the energy interval in the immediate vicinity of the Fermi level $\left(E_{F}\right)$. The divalent portion of the $\mathrm{Yb}$ \&f signal extends from $E_{F}$ to $-3 \mathrm{eV}$ in (1a) with four distinct peaks in this recion which represent the bulk and surface $4 f$ doublets for the $4 f_{7 / 2}$ and $4 f_{5 / 2}$ spin orbit components. The bulk component of the $4 f_{7 / 2}$ level is closest to $E_{F}$ and is shown in the inset of (1a) in a high resolution scan. The near Fermi level (NFL) region shown in the inset of ( la) includes the energy interval where the Kondo resonance and the CEF sidebands should be observed. In (lb) we show GS calculations for $\mathrm{YbCu} 2 \mathrm{Si} 2$ (line) which include the KR and the CEF levels determined from neutron scattering.[13] Also shown in (1b) is the GS calculation cut off by a $20 \mathrm{~K}$ Fermi function and convoluted with a $65 \mathrm{meV}$ Gaussian (diamonds) representing the experimental resolution. In (1c), this Gaussian broadened GS calculation (line) is compared to the high resolution PES data (circles), and it is clear that the calculation is not an accurate representation of the PES spectrum. In particular, the GS calculation is much narrower in energy, closer to $E_{F}$, and asymmetric in shape than the measured PES data. The parameters used for the GS calculation were; conduction band width $(\mathrm{BW})=6 \mathrm{eV}, \mathrm{CEF}$ levels at 12,30 and $80 \mathrm{meV}, \mathrm{SOS}=1.29 \mathrm{eV}$, bare f-level energy $\left(\varepsilon_{\mathrm{f}}\right)=-$ $1.0 \mathrm{eV}$ and the hybridization $(\Delta)=17.54 \mathrm{meV}$, resulting in a $T_{K}$ value of $\sim 40 \mathrm{~K}$ in agreements with thermodynamic measurements.

In order to demonstrate that the disagreement between GS calculation and PES data is not limited to one material we present PES results for three heavy fermion materials in Fig. 2 along with their associated GS calculations. The comparison for $\mathrm{YbCu} 2 \mathrm{Si} 2\left(\mathrm{~T}_{\mathrm{K}} \sim 40 \mathrm{~K}\right)$ is reproduced in (2a) while results for $\mathrm{YbAgCu} 4\left(\mathrm{~T}_{\mathrm{K}} \sim 100 \mathrm{~K}\right)$ are presented in $(2 \mathrm{~b})$ and results for $\mathrm{YbAl} 3\left(\mathrm{~T}_{\mathrm{K}} \sim 400\right.$ $\mathrm{K})$ are presented in (2c). For all three of these heavy fermions, the GS calculations (solid lines) show large discrepancies when compared with the experimental PES data (filled circles). In general, the GS calculations show a lineshape which is too narrow and too close to $\mathrm{E}_{\mathrm{F}}$ when compares with the PES experimental results. The results from Fig. 2 would indicate that the Kondo model, within the present GS framework, is not an appropriate description for the photoemission 
spectra of heavy fermions. The experimental resolution was 65,60 and $45 \mathrm{meV}$ respectively for the data of Fig. $2 \mathrm{a}-\mathrm{c}$, and the GS fitting parameters were, as stated for Fig. 1 for $\mathrm{YbCU}_{2} \mathrm{Si}_{2}$; for $\mathrm{YbAgCu}_{4} \mathrm{BW}=6 \mathrm{eV}$, no CEF levels as determined by neutron scattering[14], SOS $=1.29 \mathrm{eV}$, bare f-level energy $\varepsilon_{\mathrm{f}}=-0.75 \mathrm{eV}$, hybridization $\Delta=11.96 \mathrm{meV}$, resulting in a $T_{K}$ value of $\sim 100 \mathrm{~K}$; and for $\mathrm{YbAl}_{3} \mathrm{BW}=10 \mathrm{eV}$, no $\mathrm{CEF}$ levels, $\mathrm{SOS}=1.29 \mathrm{eV}$, bare f-level energy $\varepsilon_{\mathrm{f}}=-0.75 \mathrm{eV}$, hybridization $\Delta=14.6 \mathrm{meV}$, resulting in a $T_{\mathrm{K}}$ value of $-400 \mathrm{~K}$.

Results from non-linear least-squares lineshape analysis of four $4 \mathrm{f}$ electron materials are presented in Fig.3. On the left side of Fig. 3 we show the PES data points (circles) along with the full lineshape analysis for $\mathrm{YbCu} 2 \mathrm{Si} 2(3 \mathrm{a}), \mathrm{YbAl} 3(3 \mathrm{~b}), \mathrm{LuAl} 3(3 \mathrm{c})$, and $\mathrm{Yb}$ metal (3d). The lineshape analysis consists of two doublets for each material, one doublet representing $4 \mathrm{f}$ photoemission from the surface and another doublet representing $4 \mathrm{f}$ photoemission from the bulk. Each double consists of a SOS $4 f_{7 / 2}$ and $4 f_{5 / 2}$ pair separated by $1.29 \mathrm{eV}$. The bulk emission features are larger than the surface features and the bulk $4 \mathrm{f}_{7 / 2}$ level is centered on the zero of the relative energy scale. The lineshapes used in the analysis are Doniach-Sunjiac lineshapes, convoluted with a Gaussian to represent the experimental resolution and cut off by the appropriate Fermi function for the $\mathrm{Yb}$ materials. The energy scale for the LuAl3 data is compressed by $12 \%$ to account for the increased SOS in this material.

Although the ratios of the surface to bulk intensities varies due to differences in photon energies and divalent/trivalent $4 \mathrm{f}$ distributions for the heavy fermions, it is clear from the right side of Fig. 3 that the bulk $4 \mathrm{f}$ lineshape is very similar in all four of these materials. In particular, the fitted bulk $4 f_{7 / 2}$ natural line width is generally $-100 \mathrm{meV}(130,75,90,100 \mathrm{meV}$ from top to bottom), far greater than any predicted line width of the Kondo model, and showing surprisingly good agreement between Lu 4f levels, divalent $\mathrm{Yb}$ metal $4 \mathrm{f}$ levels, and the $4 \mathrm{f}$ levels of heavy fermions. Furthermore, these $4 \mathrm{f}$ linewidths are in good agreement with our previously reported line width of $75 \mathrm{meV}$ [15] for $\mathrm{YbAgCu}_{4}$ and this same $75 \mathrm{meV}$ line width reported for $\mathrm{YbAgCu}_{4}$ in a recent paper [11] with $20 \mathrm{meV}$ experimental resolution. These fitted natural linewidths clearly indicate the present PES study is not limited by experimental resolution, and the $4 \mathrm{f}$ linewidths are 
not consistent with the Kondo scale for the $\mathrm{Yb}$ heavy fermions. The similarity in the fitted lineshapes strongly suggests that the $4 f$ emission for the four materials included in Fig. 3 is indicative of a generalized $4 \mathrm{f}$ core-like lineshape for $4 \mathrm{f}$ materiais which is independent of the any heavy fermion characteristics.

Finally, we look at the temperature dependence of $\mathrm{Yb} 4 \mathrm{f}$ levels in an effort to better understand 4f PES photoemission and consider the large temperature variations predicted by the Kondo model for heavy fermions. Two recent photoemission papers $[9,11]$ claim to have observed variations in $\mathrm{Yb} 4 \mathrm{f}$ spectral weight with temperature which are corisistent with Kondo model NCA predictions. In ref. [5] we showed how the temperature dependence for a Ce-based heavy fermion could be fully described by simple photoemission principles including temperature dependent phonon broadening. In Fig. 4, we show Yo metal PES data for two temperature, $25 \mathrm{~K}$ (solid line) and $250 \mathrm{~K}$ (filled circles). Fig. 4a shows the raw PES data normalized to constant $4 \mathrm{f}$ signal intensity, while Fig. $4 \mathrm{~b}$ shows the bulk $4 \mathrm{f}$ component of the data fitting. In both the raw data and fitted bulk $4 \mathrm{f}$ component, the peak height of the $4 f_{7 / 2}$ peak is reduced by $21 \%$ as the temperature is increased from $25 \mathrm{~K}$ to $250 \mathrm{~K}$. Although the peak height of all four components has been reduced with temperature, the integrated area remains constant since all of the spectral features broaden with temperature. The extent of the phonon broadening is large, $130 \mathrm{meV}$ increase in the $4 \mathrm{f}_{7 / 2}$ line width ( $100 \mathrm{meV}$ natural line width at $25 \mathrm{~K}$ ) from 25 to $250 \mathrm{meV}$. Without detailed quantitative analysis, the change in peak height might be interpreted as a change in spectral weight. Moreover, high energy resolution and detailed analysis alone may not be enough to determine the nature of a $4 \mathrm{f}$ material's temperature dependence if the photoemission spectra are dominated by surface or mixed phase contributions. Specifically, only when the area of the bulk component of the $4 \mathrm{f}$ lineshape is large compared with that of the surface component, and the two components are clearly separable, (as in Figs. 3 and 4) is it possible to conduct detailed analysis with meaningful confidence limits in the fitted results. Only with a combination of high energy resolution, detailed analysis and the best possible samples obtainable is it possible to distinguish 
between simple phonon broadening which occurs in most core-levels [16], and temperature dependencies unique to the manifestation of a Kondo resonance.

\section{Conclusions}

The extent of our criticism of the Kondo model's application to heavy fermions is strictly limited to experimental PES works. Moreover, the model limitations are only tested within the framework of the GS (and associated NCA) computational methods. However, within these boundaries, we have shown that the photoemission results for Yb-based heavy fermions can be interpreted quite simply within a conventional $4 \mathrm{f}$ core-level framework. Also, for $\mathrm{Yb}$ heavy fermions, GS calculations in present form, are not in agreement with the measured PES spectra and thus not an adequate model for these materials. Finally, reported agreement between $\mathrm{Yb}$ heavy fermion temperature dependent PES $[9,11]$ and NCA predictions must make a full accounting of conventional PES temperature dependencies (as in the case of $\mathrm{Yb}$ metal) before attributing these PES results to Kondo model behavior.

This work was supported by the U.S. Department of Energy, the Synchrotron Radiation Center is operated by the University of Wisconsin for the National Science Foundation and the National Synchrotron Light Source is operated by the U.S. Department of Energy. 


\section{References}

[1] O. Gunnarsson and K. Schonhammer, Phys. Rev. B 31, 4815 (1985).

[2] N. E. Bickers, D.L. Cox and J.W. Wilkins, Phys. Rev. B 36, 2036 (1987).

[3] J.W. Allen, S.-J. Oh, O. Gunnarsson, K. Schonhammer, M.B. Maple, M.S. Torikachvili and I. Lindau, Advances in Physics 35, 275 (1986).

[4] F. Patthey, J.-M. Imer, W.-D. Schneider, H. Beck and Y. Baer, Phys. Rev. B 42, 8864 (1990)

[5] J. J. Joyce, A. J. Arko, J. M. Lawrence, P. C. Canfield, Z. Fisk, R. J. Bartlett, and J. D. Thompson, Phys. Rev. Lett. 68, 236 (1992); J.J. Joyce and A.J. Arko, Phys. Rev. Lett. 70, 1181, (1993).

[6] J.J. Joyce, A.J. Arko, J.M. Lawrence, J. Tang, P.C. Canfield, R.J. Bartlett, Z. Fisk, J.D. Thompson, and P.S. Riseborough Solid State Comm., 83, 551 (1992).

[7] J.J. Joyce et. al, Physica B 186-188, 31 (1993).

[8] R.I.R. Blyth et. al, Phys Rev. B, (in press, 1993).

[9] L.H. Tjeng et. al, Phys. Rev. Lett. (submitted).

[10] En-Jin Cho et. al, Physica B 186-188, 70 (1993).

[11] P. Weibel, M. Grioni, D. Malterre, B. Dardel, Y. Baer and M. Besnus, Z. Phys. B, (in press).

[12] S.-J. Oh et. al, Phys. Rev. B 37, 2861 (1988).

[13] R. Currat, R.G. Lloyd, P.W. Mitchell, A.P. Murani and.' W. Ross, Physica B 156\&157, 812 (1989)

[14] A. Severing, A.P. Murani,J.D. Thompson, Z. Fisk, and C.-K. Loong, Phys. Rev. B 41, 1739 (1990); C. Rossel, K.N. Yang, M.B. Maple, Z. Fisk, E. Zirngiebel and J.D. Thompson, Phys. R.ev. B 35, 1914 (1987).

[15] APS March Meeting 1992, Indianapolis, IN, 3/16-3/20/1992.

[16] D.M. Riffe, G.K. Wertheim, and P.H. Citrin, Phys. Rev. Lett. 63, 1976 (1989). 


\section{Figure Captions}

Fig. 1. Photoemission EDCs and GS calculations for the heavy fermion $\mathrm{YbCu}_{2} \mathrm{Si}_{2}$. (A) shows the full valence band with $\mathrm{Cu} 3 \mathrm{~d}$ levels $(-4$ to $-3 \mathrm{eV})$, divalent $(0$ to $-3 \mathrm{eV})$ and trivalent $(-6$ to $1 \mathrm{leV}) \mathrm{Yb} 4 \mathrm{f}$ levels and the Si $2 \mathrm{p}$ core-level $(+3.5 \mathrm{eV})$ in second order above the Fermi level. The inset for (A) shows a high resolution EDC for the near Fermi level region of $\mathrm{YbCu}_{2} \mathrm{Si}_{2}$ (B) shows a GS calculation (solid line) for $\mathrm{YbCu}_{2} \mathrm{Si}_{2}$ and the experimentally broadened calculation (diamonds) for comparison with data (60 meV Gaussian broadening). (C) shows the comparison between the experimental PES work (circles) and the GS Kondo model calculation (line), the agreement is poor as the calculation is far too narrow and close to the Fermi level.

Fig. 2. Comparison of thrce PES measurements and the associated GS Kondo model calculations; (A) $\mathrm{YbCu}_{2} \mathrm{Si}_{2},(\mathrm{~B}) \mathrm{YbAgCu}_{4}$, and $(\mathrm{C}) \mathrm{YbAl}_{3}$. The GS calculations have been cut by the appropriate Fermi function and broadened for the experimental resolution. In all cases the agreement between calculation and experiment is poor with the calculation always too narrow and close to the Fermi level.

Fig. 3. Photoemission results for the $4 f$ levels of four materials including two heavy fermions (A) $\mathrm{YbCu}_{2} \mathrm{Si}_{2}$, (B) $\mathrm{YbAl}_{3}$, the Lu counterpart (C) $\mathrm{LuAl}_{3}$ to the $\mathrm{YbAl}_{3}$, and (D) divalent $\mathrm{Yb}$ metal. The non-linear least-squares fitting to the data are also shown on the left side with the bulk $4 \mathrm{f}$ component for each material on the right side. The similarity in the bulk $4 \mathrm{f}$ lineshape indicates a generalized $4 \mathrm{f}$ lineshape independent of a material's heavy fermion characteristics.

Fig. 4. Photoemission EDCs (A) and lineshape analysis of the bulk $4 \mathrm{f}$ component (B) for divalent $\mathrm{Yb}$ metal. In both cases, the integrated area of the spectra is the same but the peak height of the bulk $4 \mathrm{f}_{7 / 2}$ level decreases by $21 \%$ going from $25 \mathrm{~K}$ (solid line) to $250 \mathrm{~K}$ (circles). The resultant loss in amplitude is made up by increased line width due to phonon broadening as the temperature of the $\mathrm{Yb}$ is increased. The line width increases by $130 \mathrm{meV}$ between $25 \mathrm{~K}$ and $250 \mathrm{~K}$. 


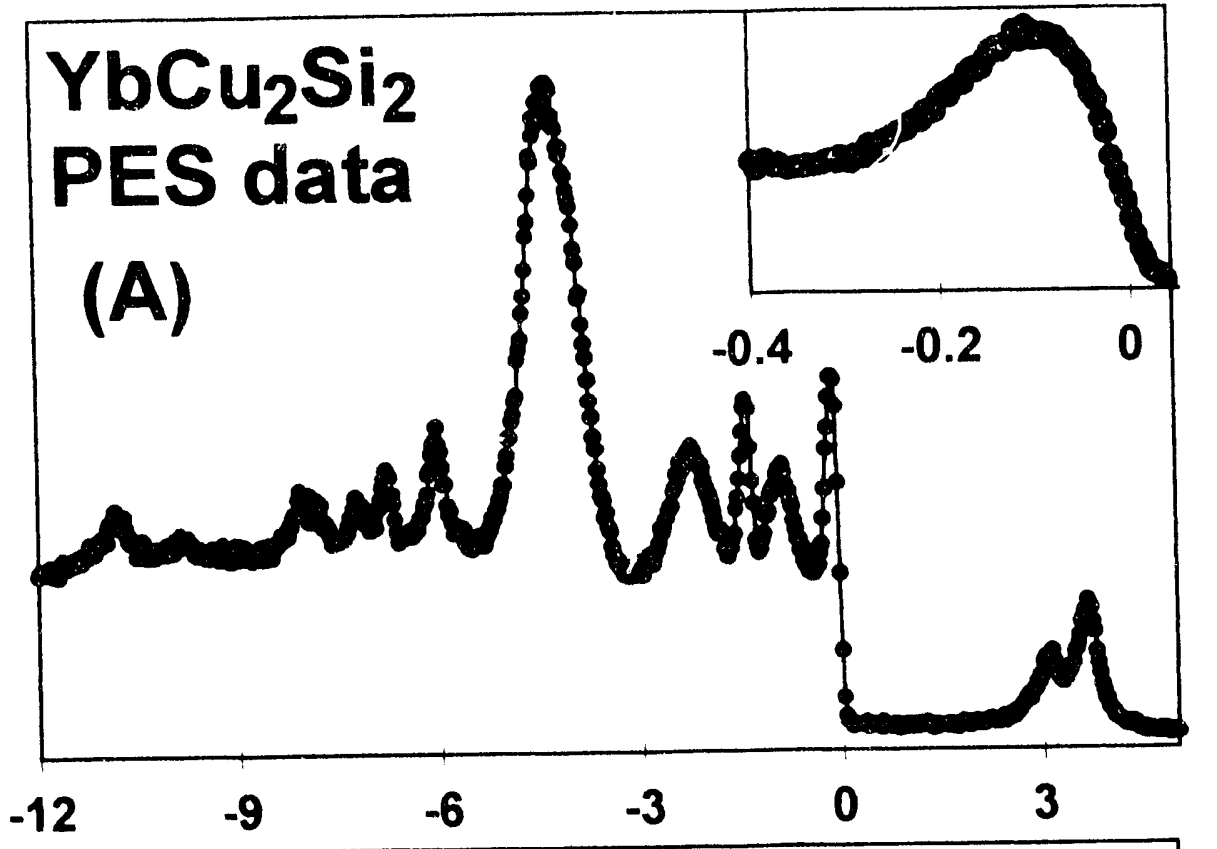

$\mathrm{YbCu}_{2} \mathrm{Si}_{2}$

GS calculation

(B)
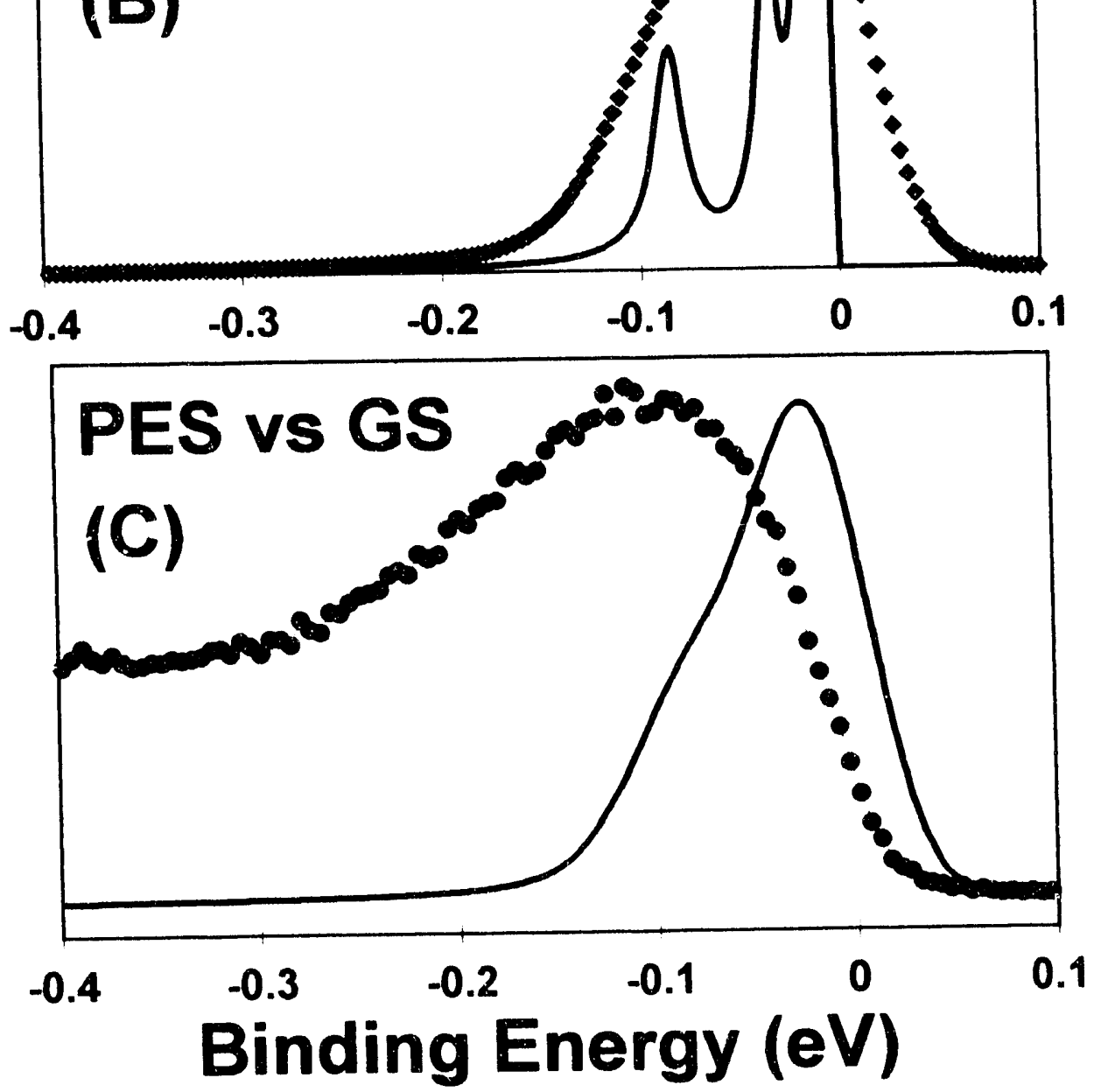

Fig. 1 


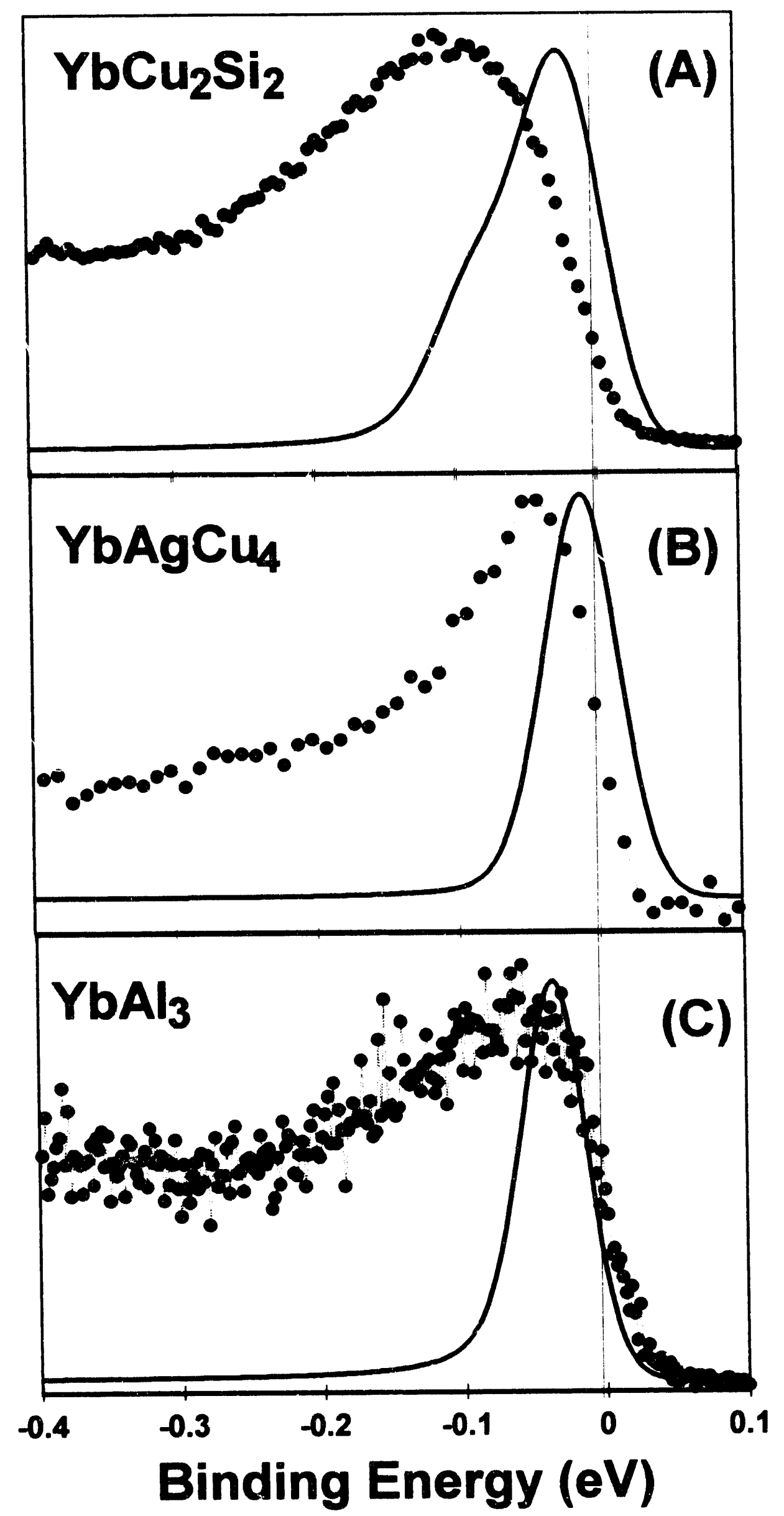

Fig. 2 


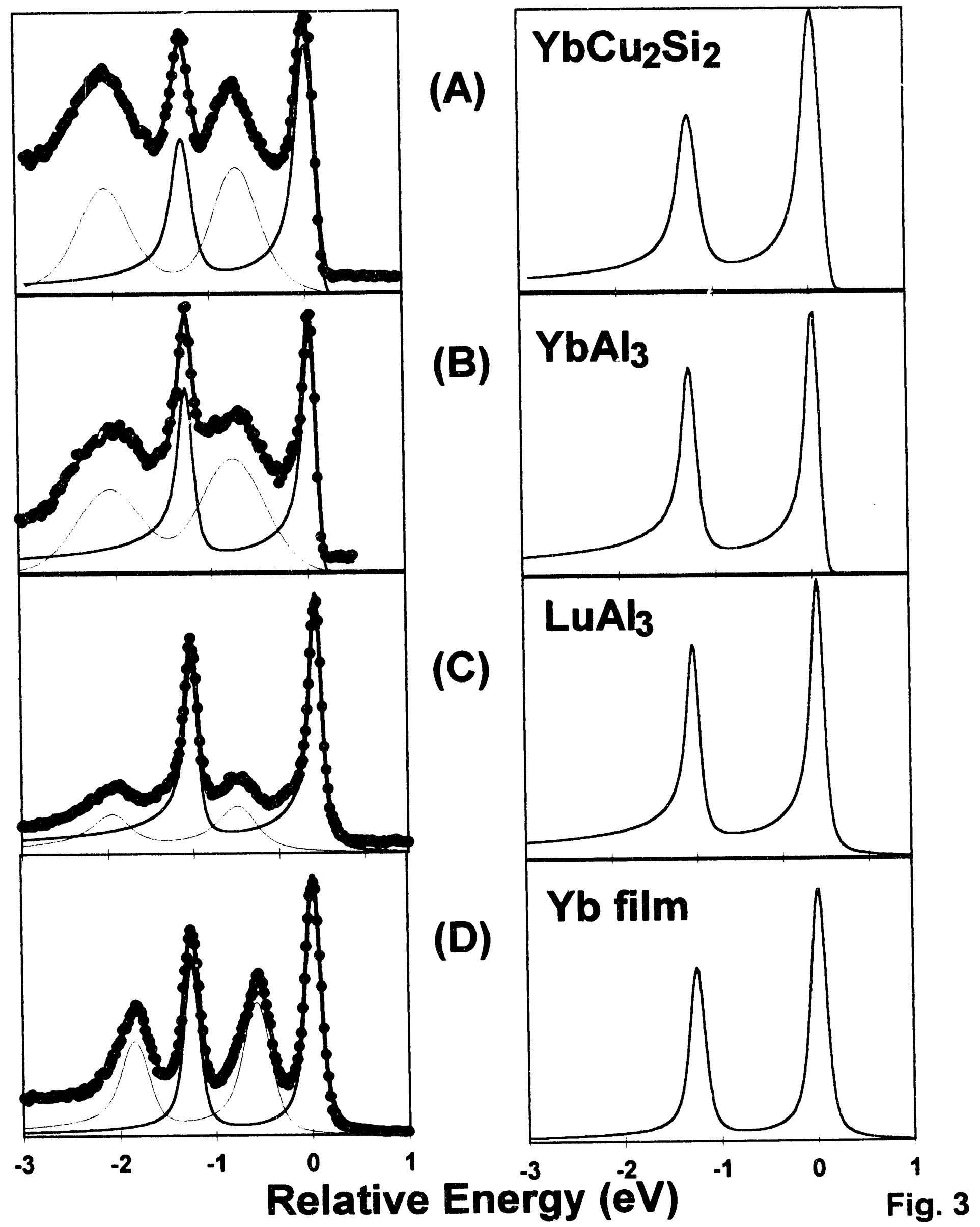



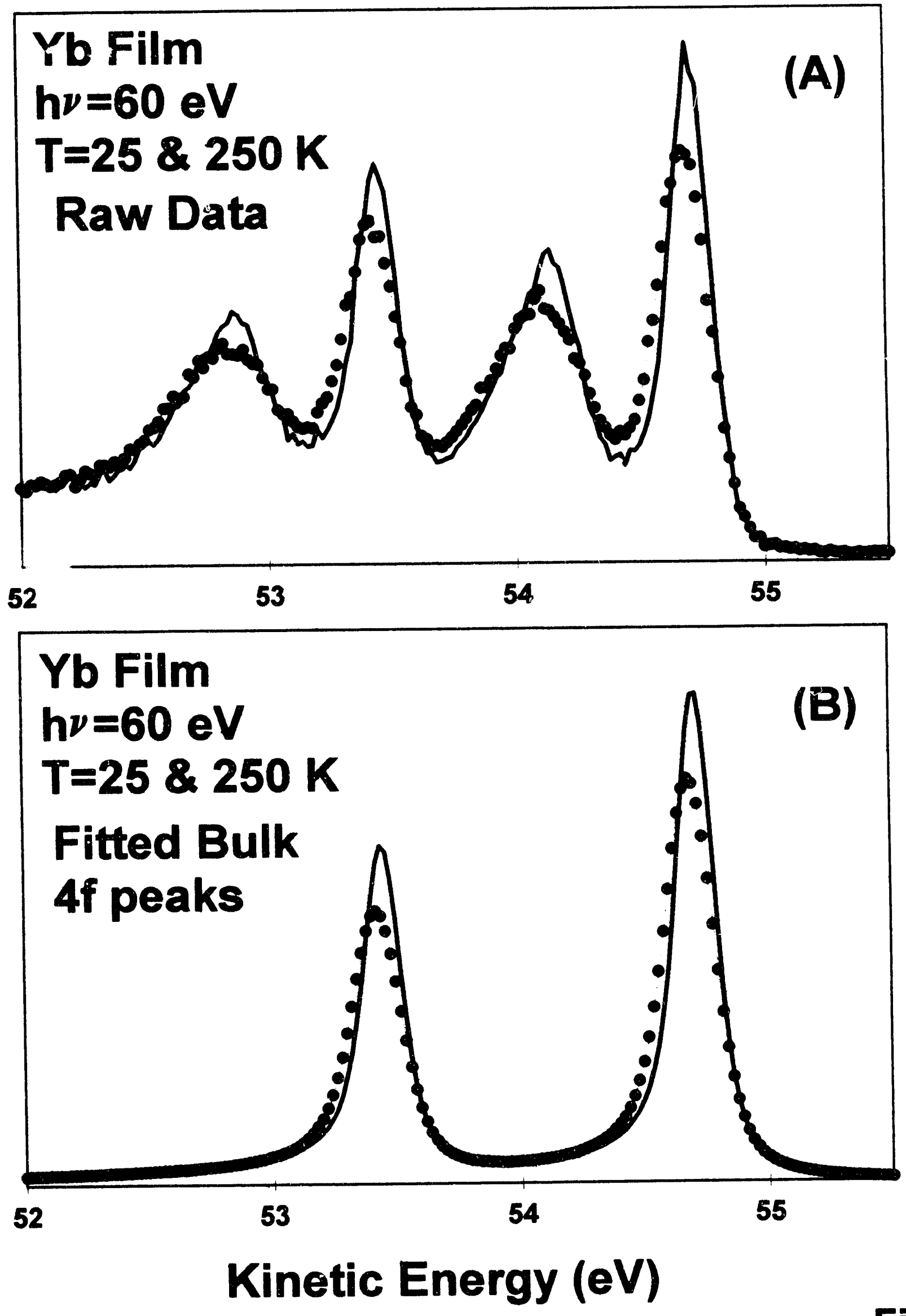

Fig. 4 

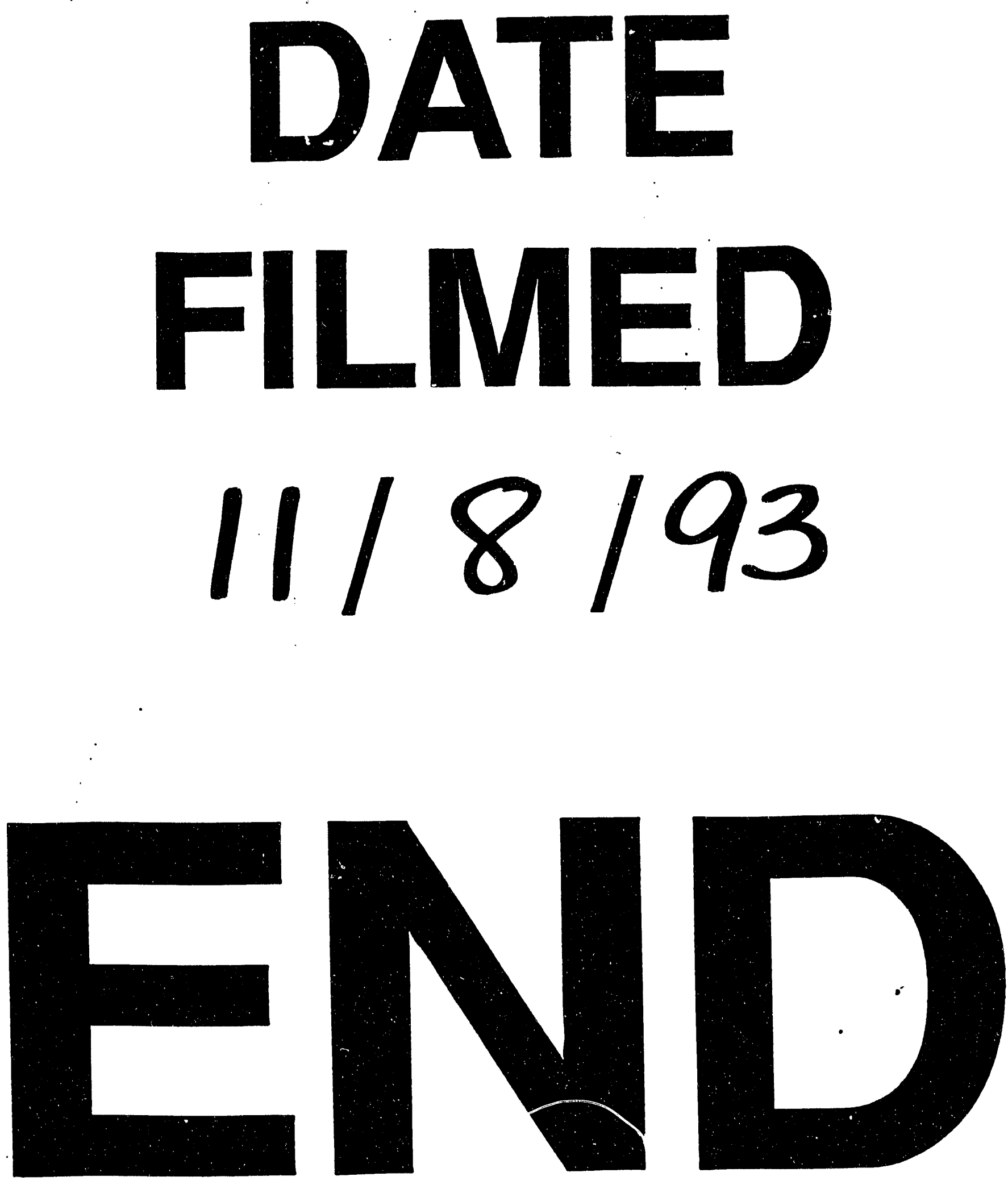


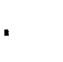

